\title{
Serum cytokines correlated with the disease severity of generalized pustular psoriasis
}

\author{
Masaaki Yamamoto ${ }^{1}$, Yasutomo Imai ${ }^{1}$, Yoshiko Sakaguchi, Takashi Haneda and Kiyofumi Yamanishi* \\ Department of Dermatology, Hyogo College of Medicine, Hyogo, Japan
}

\begin{abstract}
To characterize serum biomarkers reflecting the severity of generalized pustular psoriasis (GPP), we measured multiple cytokine/chemokine levels in 39 serum samples from 6 cases with GPP during the course of the disease. Serum levels of IL-4, IL-8, CXCL1 and CCL3 were positively correlated with the severity scores of GPP, white blood cell counts and serum C-reactive protein levels. Serum levels of IL-1 $\beta$, IL-1ra, IL-6, IL-10, IL-12p70, IL-18, IL-22, IFN- $\gamma$ and VEGF showed strong positive correlations $(r>0.4, p<0.01)$ with all those 3 clinical markers. Of those, IL-10 and IL-22 were significantly decreased after treatment in parallel with the GPP score and therefore those two serum cytokines might be useful to evaluate the efficacy of treatment for GPP.
\end{abstract}

Keywords: Generalized pustular psoriasis, cytokine, chemokine, serum, biomarker, severity of illness index

\section{Introduction}

Psoriasis is a chronic inflammatory skin disease characterized by erythematous papules and plaques with thick scales. Psoriasis is an immune-mediated disease because it can be effectively treated with anti-p40 subunit of IL-12/23 or with anti-TNF- $\alpha$ [1]. Psoriasis is classified in 5 subtypes, psoriasis vulgaris (PV), psoriasis guttata, psoriatic arthritis, psoriatic erythroderma and generalized pustular psoriasis (GPP). Of those subtypes, GPP is a rare and severe form of psoriasis, in which acute generalized erythema and scaling develop with a rapid spread of multiple sterile pustules and is often associated with fever and general fatigue. During the clinical course of GPP with frequent recurrence, laboratory tests show leukocytosis and elevated levels of C-reactive protein (CRP). Patients with GPP are often complicated by arthritis, respiratory failure and ocular symptoms such as iritis, uveitis and conjunctivitis. Besides the skin manifestations, the severity of GPP has also been evaluated using blood tests, such as white blood cell count (WBC), CRP and serum albumin lev-

\footnotetext{
${ }^{1}$ M. Y. and Y. I. contributed equally to this work.

* Corresponding author: Kiyofumi Yamanishi, Department of Dermatology, Hyogo College of Medicine, 1-1, Mukogawa-cho, Nishinomiya, Hyogo 663-8501, Japan. Tel.: +81 79845 6653; Fax: +81 79845 6651, E-mail: kyamanis@hyo-med.ac.jp.
}

els. However, those serum markers do not necessarily reflect the disease activity of GPP, and therefore, more specific markers are required for the proper evaluation of this disease.

In patients with $\mathrm{PV}$, several studies have shown that serum levels of IL-6, IL-7, IL-8, IL-12p70, IL-17, IL18 , TNF- $\alpha$, IFN- $\gamma$ and VEGF are increased [2-5]. On the other hand, only serum levels of IFN- $\gamma$ and IL-6 have been examined in GPP. The serum levels of IFN- $\gamma$ are higher in the serum of patients with GPP than in healthy controls [6]. Nakamura et al. [7] have reported that serum IL-6 levels were high in a 6-year-old boy with GPP and it decreased as the erythema improved by treatment with cyclosporine.

In this study, we characterized levels of multiple serum cytokine and chemokine markers in 6 patients with GPP and assessed their correlations with GPP scores, WBC and CRP levels during the course of the disease.

\section{Patients and methods}

\subsection{Cases}

Six cases with GPP as listed below were enrolled in this study. This study was approved by the Ethical Committee of the Hyogo College of Medicine, and informed consent was obtained from all subjects. 
Table 1

Diagnosis criteria of severity classification for GPP

A. Evaluation of skin symptoms (0-9)

\begin{tabular}{lccc}
\hline & Severe & Moderate & Mild \\
\hline Erythema coverage (overall) $^{*}$ & 3 & 2 & 1 \\
Erythema coverage with pustule* $^{* *}$ & 3 & 2 & 0 \\
Edematous erythema coverage** $^{* *}$ & 3 & 2 & 0 \\
\hline
\end{tabular}

$* \%$ of the total body surface area (Severe: $75 \%$ or more, Moderate: between 25 and $75 \%$, Mild: below $25 \%$ );

**\% of the total body surface area (Severe: $50 \%$ or more, Moderate: between 10 and 50\%, Mild: below $10 \%$ ).

B. Evaluation of overall symptoms and examination results $(0-8)$

\begin{tabular}{llll}
\hline Score & 2 & 1 & \\
\hline Fever $\left({ }^{\circ} \mathrm{C}\right)$ & 38.5 or above & Between 37 and 38.5 & Below 37 \\
White blood cell counts & 15,000 or above & Between 10,000 and 15,000 & Below 10,000 \\
CRP & 7.0 or above & Between 0.3 and 7.0 & Below 0.3 \\
Serum albumin & Below 3.0 & Between 3.0 and 3.8 & 3.8 or above \\
\hline
\end{tabular}

Severity classification (Total scores): Mild (0-6); Moderate (7-10); Severe (11-17).
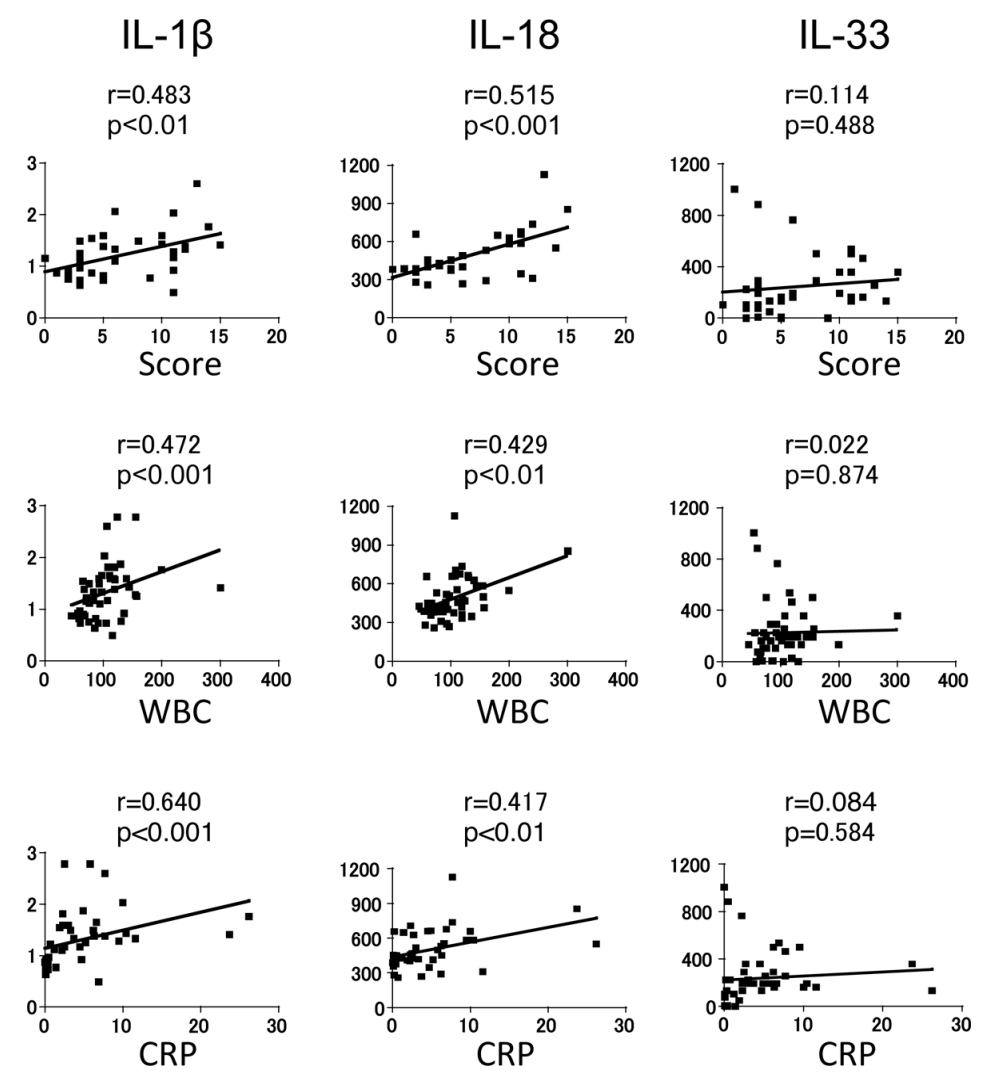

Fig. 1. Correlations of serum levels of IL-1 family cytokines with the GPP score, WBC and CRP levels. Serum levels of IL- $1 \beta$ and IL- 18 in GPP cases showed a positive correlation with all those clinical markers. Units for cytokines, WBC and CRP levels are pg/mL, $\times 100 / \mathrm{mm}^{3}$ and $\mathrm{mg} / \mathrm{dL}$, respectively. A linear regression line is indicated in each graph. The correlation coefficient (r) was calculated by Spearman's test. A $P$-value of less than 0.05 is considered statistically significant.

\section{Case 1}

A 73 year-old man, who complained of repeated exacerbation of GPP since 1990, had been treated with $100 \mathrm{mg}$ cyclosporine daily. He had a past history of hepatitis B and lung tuberculosis. In March 2010, acute exacerbation of GPP recurred with a fever of over $39^{\circ} \mathrm{C}$. His disease has been effectively controlled by infliximab at $5 \mathrm{mg} / \mathrm{kg}, 14$ times in total, as of April 2012. Reactivation of hepatitis B or tuberculosis did not occur during that time. 

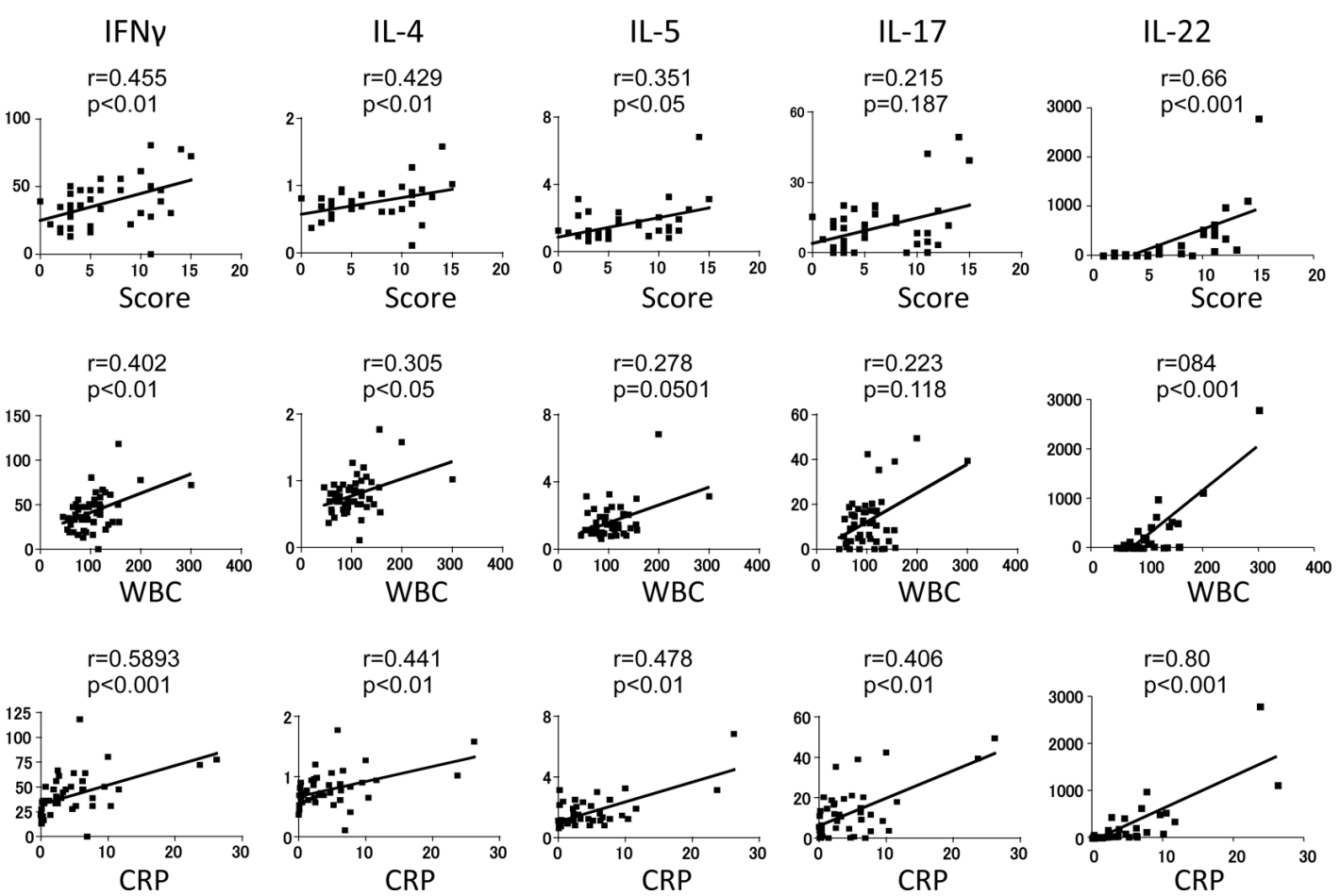

Fig. 2. Correlation of serum levels of cytokines produced by T-cells with the GPP score, WBC and CRP level. Serum levels of IFN- $\gamma$ (a Th1 cytokine), IL-4 (a Th2 cytokine) and IL-22 (a Th17 cytokine) in GPP cases showed a positive correlation with all those clinical markers. Units for cytokines, WBC and CRP levels are $\mathrm{pg} / \mathrm{mL}, \times 100 / \mathrm{mm}^{3}$ and $\mathrm{mg} / \mathrm{dL}$, respectively. A linear regression line is indicated in each graph. The correlation coefficient ( $\mathrm{r}$ ) was calculated by Spearman's test. A $P$-value of less than 0.05 is considered statistically significant.

\section{Case 2}

A 55 year-old man, who had PV since 1988, visited our Department in February 2002, with generalized erythema and multiple pustules on his entire body with a fever. He had a 20 year-history of diabetes and high blood pressure, and had smoked for 40 years. The histopathology of a lesion was compatible with GPP. He had been treated with prednisolone and cyclosporine, but his symptoms were unstable. Treatment with a combination of $125 \mathrm{mg}$ cyclosporine daily, $4 \mathrm{mg}$ methotrexate (MTX) weekly and $5 \mathrm{mg}$ prednisolone daily was effective, and after 4 years of the treatment, there has been no flare up of GPP to date.

\section{Case 3}

A 70 year-old man complained of a fever of $39^{\circ} \mathrm{C}$ and erythema with pustules on his entire body except his face. He had a 40 year-history of alcoholism, but no previous history of psoriasis. A skin biopsy showed Kogoj's spongiform pustules. He was treated with $40 \mathrm{mg}$ etretinate daily and $12.5 \mathrm{mg}$ prednisolone daily since November 2006, and his skin eruptions resolved in 8 months.

\section{Case 4}

A 55 year-old man, who complained of $\mathrm{PV}$, had been treated with corticosteroids since 1989. In early 2004, erythema with pustules and scales appeared on his entire body. He had smoked from his youth and had a history of stomach cancer in 1994. Treatment with oral $40 \mathrm{mg}$ etretinate daily with topical tacalcitol lotion was effective. A recurrence of GPP happened after he withdrew from those drugs in 2006, but has been controlled by restarting the etretinate.

\section{Case 5}

A 63 year-old woman with PV had been treated with topical tacalcitol and/or corticosteroids since 1974. She had about a 10 year-history of rheumatic polymyalgia and had been treated with $5 \mathrm{mg}$ prednisolone daily. In July 2007, she visited our Department because of the exacerbation of her skin eruptions. Oral cyclosporine was not effective and diffuse erythema with scale and pustules appeared with a fever of $38.7^{\circ} \mathrm{C}$ in a few months. Cyclosporine was replaced with $6 \mathrm{mg}$ MTX weekly, and her skin eruptions and fever were improved in 3 weeks. 

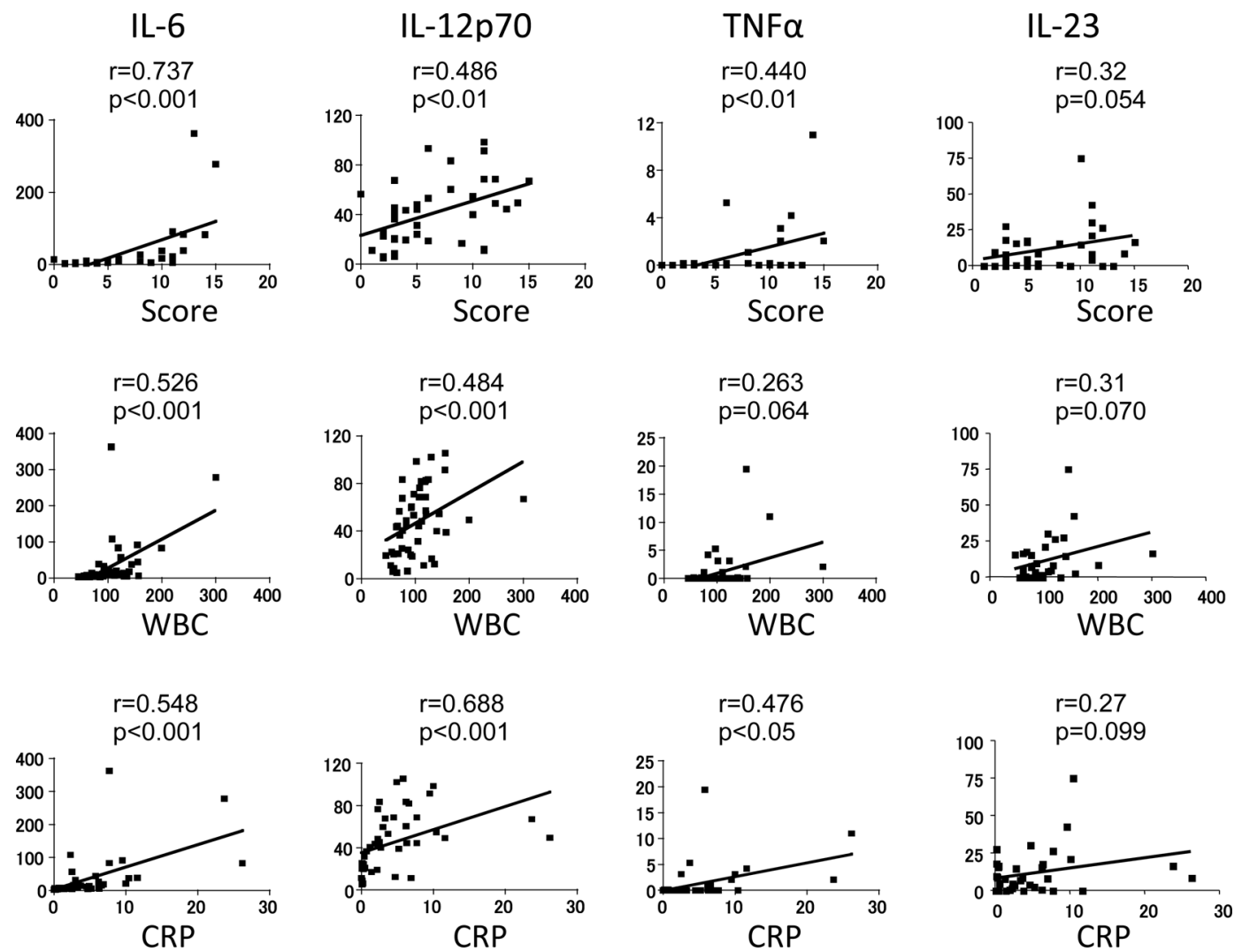

Fig. 3. Correlation of serum levels of cytokines produced by monocytes with the GPP score, WBC and CRP level. Serum levels of IL-6 and IL-12p70 in GPP cases showed a significant correlation with all of those 3 clinical markers. Units for cytokines, WBC and CRP are $\mathrm{pg} / \mathrm{mL}$, $\times 100 / \mathrm{mm}^{3}$ and $\mathrm{mg} / \mathrm{dL}$, respectively. A linear regression line is indicated in each graph. The correlation coefficient (r) was calculated by Spearman's test. A $P$-value of less than 0.05 is considered statistically significant.

\section{Case 6}

A 58 year-old man complained of generalized diffuse erythema with pustules in February 2006. He had PV and had been treated with topical betamethasone butyrate propionate. He had a past history of chronic hepatitis B since 1992 and high blood pressure since 2005. He also had smoked for 31 years. A diagnosis of GPP was made from a skin biopsy and he was effectively treated with topical tacalcitol.

\subsection{ELISA and protein array}

Serum concentrations of IL- $1 \beta$, IL-1ra, IL-4, IL-5, IL-6, IL-7, IL-8, IL-10, IL-12p70, IL-17, IFN- $\gamma$, CXCL10 (IP-10), CCL2 (MCP-1), CCL3 (MIP-1 $\alpha$ ), CCL4 (MIP-1 $\beta$ ), CCL5 (RANTES), CCL11 (Eotaxin), TNF$\alpha$ and VEGF were measured using Bio-Plex Pro human cytokine multiplex assay kits (Bio-Rad, Hercules, CA) and a Bio-Plex 200 System with high-throughput fluidics. The serum concentration of IL-18 was deter- mined using a Human IL-18 ELISA kit (MBL, Tokyo, Japan). Serum concentrations of CXCL1 (GRO- $\alpha$ ), IL22 and IL-23 were determined using a Quantikine Human ELISA kit (R\&D, Minneapolis, MN). The serum concentration of IL-33 was determined by ELISA as previously described [8].

\subsection{Scoring of GPP}

The GPP score in each case was calculated using the criteria of severity classification for GPP (Table 1), which was reported by "the research team on rare intractable skin diseases" of Japan [9].

\subsection{Statistics}

The timing of blood sampling was at the baseline or at the first visit (before therapy) and at follow-ups after the treatment of patients, but blood sampling was not necessarily scheduled, because additional samplings of 

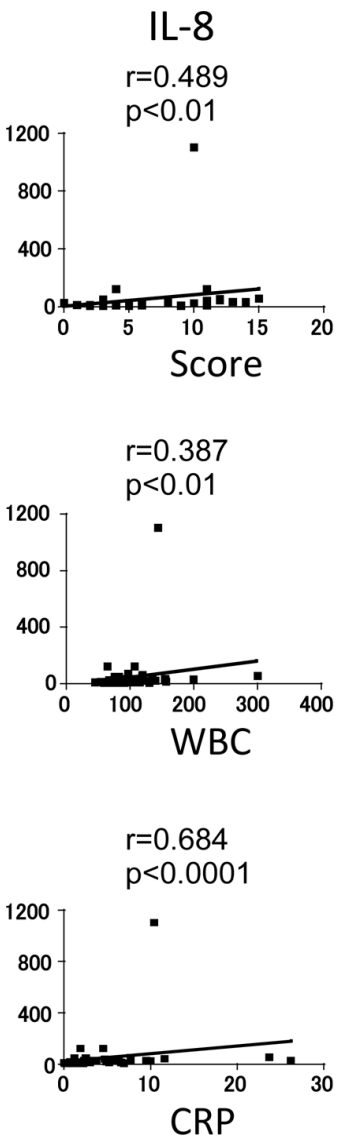

CXCL1

$r=0.433$

$\mathrm{p}<0.01$

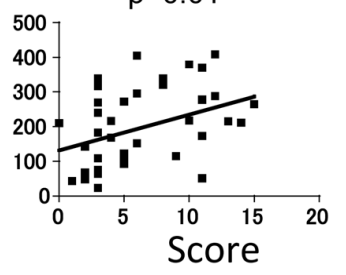

$r=0.355$

$p<0.05$

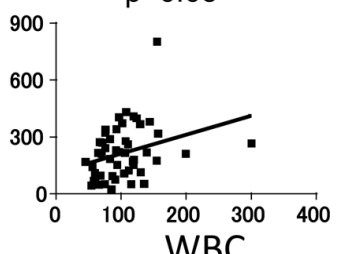

$r=0.622$

$p<0.0001$

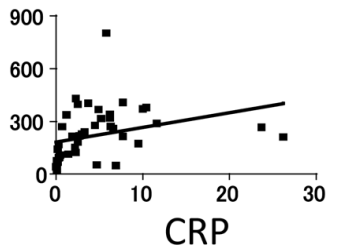

Fig. 4. Correlation of serum levels of chemokines which induce neutrophils with the GPP score, WBC and CRP level. Serum levels of CXCL1 and IL-8 in GPP cases showed a positive correlation with all those clinical markers. Units for cytokines, WBC and CRP are $\mathrm{pg} / \mathrm{mL}, \times 100 / \mathrm{mm}^{3}$ and $\mathrm{mg} / \mathrm{dL}$, respectively. A linear regression line is indicated in each graph. The correlation coefficient (r) was calculated by Spearman's test. A $P$-value of less than 0.05 is considered statistically significant.

human materials except for clinical need were not permitted by the Ethical Committee. Data were analyzed using GraphPad Prism version 4 (GraphPad Software, Inc, San Diego, CA). Correlations were calculated using Spearman's test. A $P$-value less than 0.05 is considered statistically significant.

\section{Results}

Sera were obtained from 6 patients with GPP (5 males and 1 female; age, 55-77 years; mean, 66 years old), who visited the Department of Dermatology at the Hyogo College of Medicine, from 2006 to 2009. During therapy, a total of 39 serum samples were collected from those patients, the concentrations of mul-
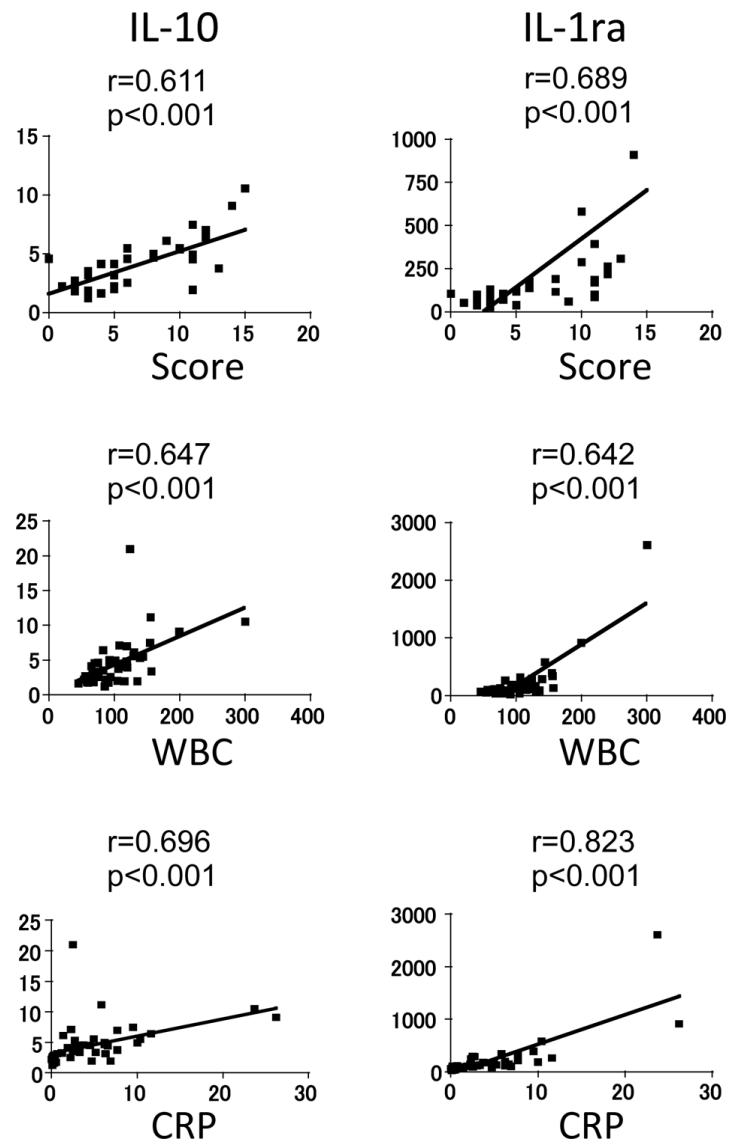

Fig. 5. Correlation of serum levels of immunosuppressive cytokines with the GPP score, WBC and CRP level. Serum levels of IL-10 and IL-ra in GPP cases showed a significant correlation with all those clinical markers. Unit for cytokines, WBC and CRP are $\mathrm{pg} / \mathrm{mL}, \times$ $100 / \mathrm{mm}^{3}$ and $\mathrm{mg} / \mathrm{dL}$, respectively. A linear regression line is indicated in each graph. The correlation coefficient (r) was calculated by Spearman's test. A $P$-value of less than 0.05 is considered statistically significant.

tiple cytokines and chemokines were measured, and their correlations with the severity score of GPP, WBC and CRP levels were statistically assessed. GPP is a rare disease and therefore, it was practically difficult to enroll a sufficient number of patients to standardize the baseline activity of the disease. In the present study, we evaluated serum levels of the following cytokines and chemokines: IL-1 $\beta$, IL-1ra, IL-4, IL-5, IL-6, IL-7, IL-8, IL-10, IL-12p70, IL-17, IL-18, IL-22, IL-23, IL33, IFN- $\gamma$, CXCL1 (GRO- $\alpha$ ), CXCL10 (IP-10), CCL2 (MCP-1), CCL3 (MIP-1 $\alpha$ ), CCL4 (MIP-1 $\beta$ ), CCL5 (RANTES), CCL11 (Eotaxin), TNF- $\alpha$, and VEGF. The increase in serum levels of IL-1 $\beta$, IL-1ra, IL-6, IL-10, IL-12p70, IL-18, IL-22, IFN- $\gamma$ and VEGF were strongly correlated $(r>0.4, p<0.01)$ with GPP score, WBC and CRP levels. 


\subsection{IL-1 family}

The IL- 1 family members, IL-1 $\beta$, IL-18 and IL-33, are proinflammatory cytokines which are produced by a variety of cells, such as epithelial cells including epidermal keratinocytes $[10,11]$. As shown in Fig. 1, the serum levels of IL- $1 \beta$ and IL- 18 in the 6 GPP cases showed positive correlations with the GPP score, WBC and CRP levels. In contrast, none of those 3 clinical markers correlated with serum IL-33 levels in the GPP patients.

\subsection{Cytokines produced by T-cells}

The serum levels of IFN- $\gamma$, a T-helper 1 (Th1) cytokine, showed a significant correlation with the GPP score, WBC and CRP levels in our GPP cases (Fig. 2). Unexpectedly, the serum levels of IL-4 and IL-5, which are Th2 cytokines, showed positive correlations with those 3 clinical markers. IL-17 and IL-22 are produced by Th17 cells infiltrating the psoriatic lesions [12]. Serum IL-17 levels in the 6 GPP cases showed a correlation only with CRP, while IL-22 showed a significant correlation with all 3 of those clinical markers.

\subsection{Cytokines produced by monocytes}

Serum IL-6 and IL-12p70 levels showed significant correlations with the GPP score, WBC and CRP levels (Fig. 3). Serum TNF- $\alpha$ levels were correlated with the GPP score and CRP, although the concentrations of TNF- $\alpha$ were very low and undetectable in some serum samples. IL-23 induces the differentiation of naïve $\mathrm{T}$ cells to Th17 cells, but none of those clinical markers was correlated with serum levels of IL-23.

\subsection{Chemokines recruiting neutrophils}

Serum CXCL1 and IL-8 levels showed positive correlations with the GPP score, WBC and CRP levels (Fig. 4).

\subsection{Immunosuppressive cytokines}

Serum IL-10 and IL-1ra levels also showed significant correlations with the GPP scores, WBC and CRP levels (Fig. 5).

\subsection{Growth factors}

Serum VEGF levels were significantly correlated with the GPP scores, WBC and CRP levels (Fig. 6). On
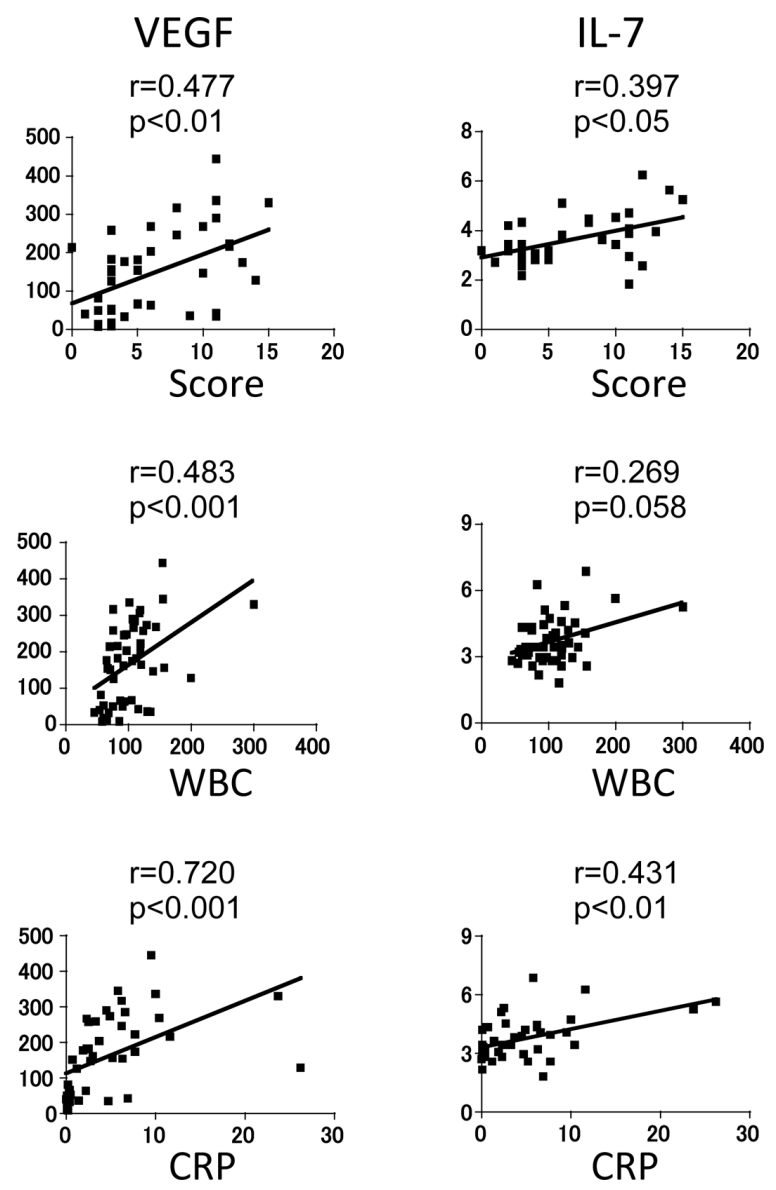

Fig. 6. Correlation of serum levels of growth factors with the GPP score, WBC and CRP level. Serum VEGF levels in GPP cases were significantly correlated with all those clinical markers. Units for cytokines, WBC and CRP are $\mathrm{pg} / \mathrm{mL}, \times 100 / \mathrm{mm}^{3}$ and $\mathrm{mg} / \mathrm{dL}$, respectively. A linear regression line is indicated in each graph. The correlation coefficient (r) was calculated by Spearman's test. A $P$-value of less than 0.05 is considered statistically significant.

the other hand, serum IL-7 levels showed a significant correlation with two of them, the GPP score and CRP.

\subsection{Th1 chemokines}

Serum CXCL10 levels showed a significant correlation with the GPP score and CRP level. Serum CCL3 levels showed a positive correlation with all 3 of those clinical markers, the GPP score, WBC and CRP level. On the other hand, none of those 3 clinical markers correlated with serum CCL2 or CCL4 levels (Fig. 7).

\subsection{Th2 chemokines}

Serum CCL11 and CCL5 levels showed negative correlations with the GPP scores and CRP levels, and 

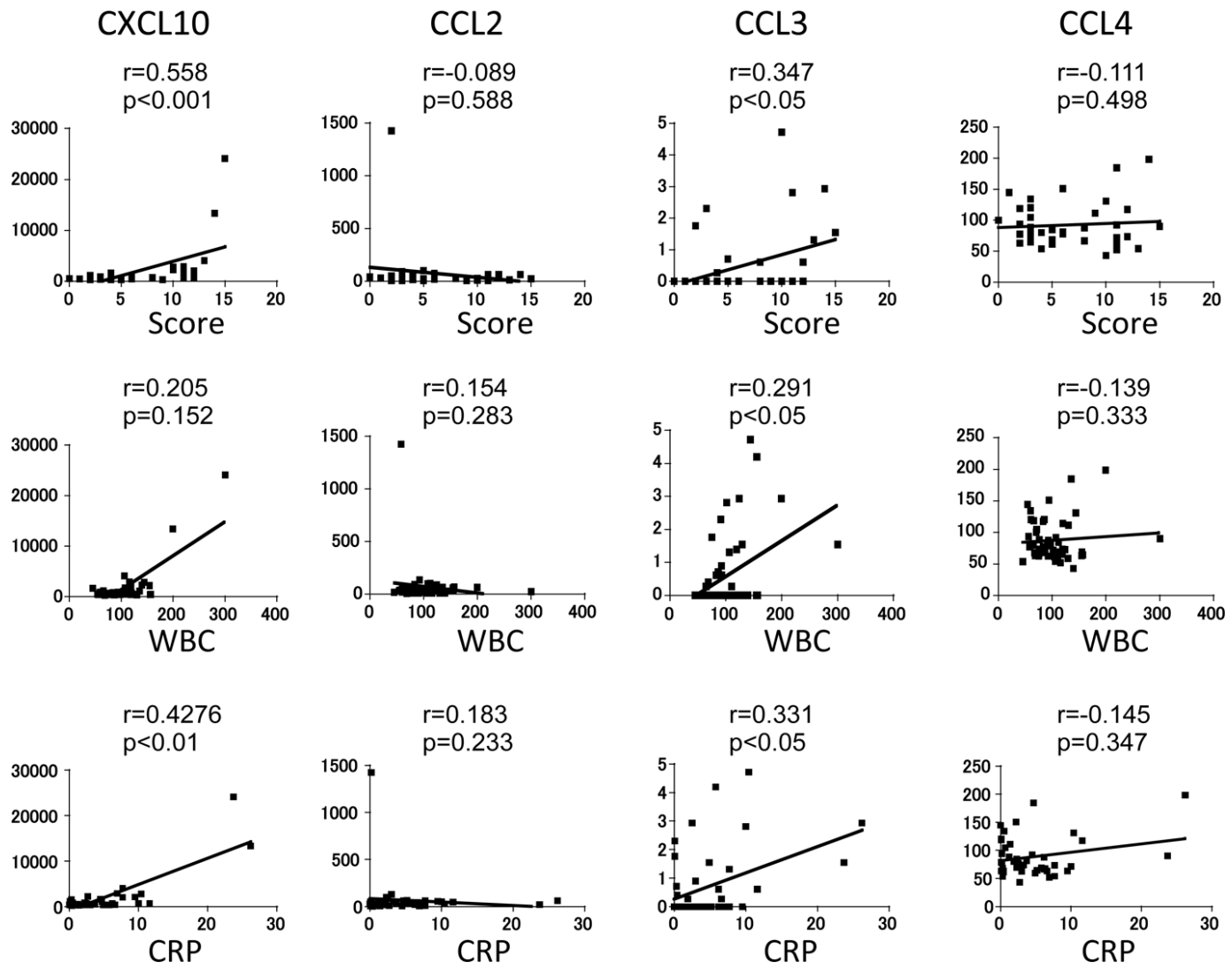

Fig. 7. Correlation of serum levels of Th1 chemokines with the GPP score, WBC and CRP level. Serum levels of CCL3 in GPP cases showed a positive correlation with all those clinical markers. Units for cytokines, WBC and CRP are $\mathrm{pg} / \mathrm{mL}, \times 100 / \mathrm{mm}^{3} \mathrm{and} \mathrm{mg} / \mathrm{dL}, \mathrm{respectively}$. A linear regression line is indicated in each graph. The correlation coefficient (r) was calculated by Spearman's test. A $P$-value of less than 0.05 is considered statistically significant.

with the GPP score and WBC, respectively (Fig. 8). Of those, CCL11 showed a strong negative correlation with the GPP score. No chemokines/cytokines other than CCL11 and CCL5 showed a negative correlation with those clinical markers in this study.

\subsection{Serum IL-10 and IL-22 before and after treatment}

Among the cytokines/chemokines that correlated with the severity score of GPP, IL-10 and IL-22 showed both high concentrations in the serum and a high correlation coefficient with the GPP score. Furthermore, as shown in Fig. 9, the levels of those cytokines were significantly decreased in parallel with the GPP score before and after treatment in the 6 patients with GPP.

\section{Discussion}

In the present study, we studied a wide range of serum cytokine/chemokine profiles in the course of GPP and found that serum levels of IL-4, IL-7, IL-8, IL-17, CXCL1, CXCL10, CCL3 and TNF- $\alpha$, and more strongly, IL-1 $\beta$, IL-1ra, IL-6, IL-10, IL-12p70, IL-18, IL$22, \mathrm{IFN}-\gamma$ and VEGF, are positively correlated with the severity scores of the disease, with WBC and with serum CRP levels. The WBC count and CRP value are included in the GPP score used in this study (Table 1) and therefore, it was difficult to compare whether WBC, CRP or a serum chemokine/cytokine is more correlated with the severity of GPP. Of those, IL- $1 \beta$, IL8, IL-12p70, IL-18, IL-22, CXCL1, IFN- $\gamma$ and VEGF are increased in patients with PV [4]. In PV, serum IL10 levels are low before treatment and are negatively correlated with the Psoriasis Area and Severity Index (PASI), and those are reversed after treatment [4,13]. In contrast, the present study reveals that the serum levels of IL-10 are positively correlated with the severity of GPP and are in parallel with the GPP score, before and after treatment. IL-1ra, IL-4 and IL-10 can attenuate an excessive Th1-type reaction via the suppression of 

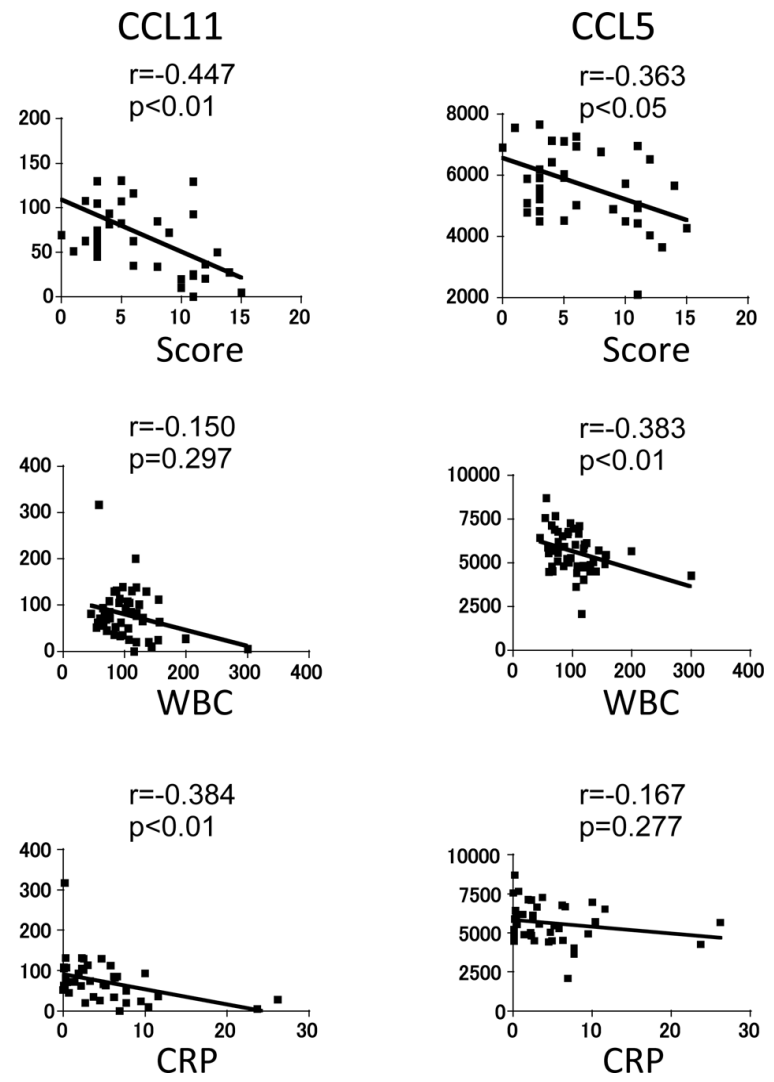

Fig. 8. Correlation of serum levels of Th2 chemokines with the GPP score, WBC and CRP level. Serum levels of CCL11 and CCL5 in GPP cases showed a negative correlation with all those clinical markers. Units for cytokines, WBC and CRP are $\mathrm{pg} / \mathrm{mL}, \times 100 / \mathrm{mm}^{3}$ and $\mathrm{mg} / \mathrm{dL}$, respectively. A linear regression line is indicated in each graph. The correlation coefficient (r) was calculated by Spearman's test. A $P$-value of less than 0.05 is considered statistically significant.

pro-inflammatory cytokines such as IL-6 that are released from activated macrophages [14]. Those Th2type cytokines might be induced as a negative feedback to regulate the generalized severe inflammatory condition in GPP. Indeed, IL-10 is up-regulated with cytokines, including IL-23 and VEGF, in other inflammatory diseases, such as age-related macular degeneration [15] and Behçet's disease [16]. Alternatively, the increase in the serum level of IL-10 might reflect an urgent angiogenesis in GPP, because IL-10 has an angiogenic activity [15].

Besides Th1 cells, Th17 cells that produce IL-17 and IL-22 are a recently identified CD4-positive T-cell subset, which has been implicated in the pathogenesis of psoriasis [12]. In psoriasis, IL-23 released from dermal TNF- $\alpha$ iNOS producing dendritic cells (dermal Tip-DC) directly induce the production of IL-17 and IL-22 from Th17 cells [17]. IL-23 is a heterodimer

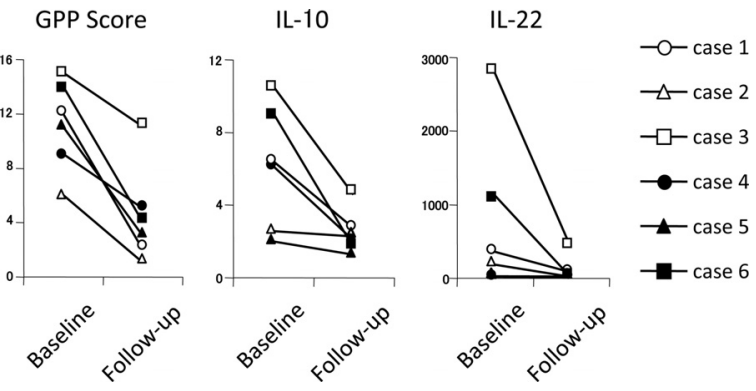

Fig. 9. GPP scores and serum IL-10 and IL-22 levels before and after treatment. The GPP scores and serum concentrations of IL-10 or IL-22 before treatment are shown at Baseline; those after treatment are shown at Follow-up.

of p19 and p40 subunits, and IL-23p19 mRNA levels are increased in psoriatic skin. Toichi et al. reported that a blockade of IL-12p40 using ustekinumab downregulated IL-12p40 and IL-23p19 mRNA levels in lesional skin [13]. Murakami et al. showed that serum levels of IL-17 and IL-22 are significantly increased in palmoplantar pustulosis (PPP), a localized form of pustular psoriasis, however, the serum level of IL-23 is not increased [18]. Low levels of serum IL-23 were also found in our cases with GPP, therefore, IL-23 might be maintained at a low level in the serum. Recently, Yilmaz et al. [19] reported that the IL-17 levels were significantly higher in the pustular psoriasis group than in the healthy controls. In this study, serum IL-17 levels were significantly correlated with CRP levels, but not with the GPP score. On the other hand, IL-22 showed a significant correlation with the GPP score and decreased with the GPP score after treatment. Recently, Lo et al. [20] reported that serum levels of IL-22 correlate with the PASI score. Hence, IL-22 may be a candidate serum biomarker that reflects the skin severity in GPP as well as in PV.

The limitations of this study are as follows: the number of samples was small and baseline disease severity was variable. In conclusion, serum levels of IL- $1 \beta$, IL1ra, IL-6, IL-10, IL-12p70, IL-18, IL-22, IFN- $\gamma$ and VEGF might reflect the clinical severity of GPP, and among those, IL-10 and IL-22 might be useful to evaluate the efficacy of treatment for GPP, although the findings of this study should be confirmed in a prospective study focusing on IL-10 and IL-22 levels in a larger number of patients.

\section{Acknowledgements}

The authors thank members of the Joint-Use Research Facilities of the Hyogo College of Medicine for 
their technical assistance. This work was supported in part by MEXT (or JSPS) KAKENHI (22791093, 23591661, 23791297 and 24791183), by a High-Tech Research Center Grant, and by a grant from the "Research on Measures for Intractable Diseases" Project: matching fund subsidy (H23-028) from Ministry of Health, Labour and Welfare, Japan.

\section{Conflict of interest}

The authors have no financial conflict of interest.

\section{References}

[1] A. Menter and C.E. Griffiths, Current and future management of psoriasis, Lancet 370 (2007), 272-284.

[2] J.C. Szepietowski, E. Bielicka, P. Nockowski, A. Noworolska and F. Wasik, Increased interleukin-7 levels in the sera of psoriatic patients: lack of correlations with interleukin-6 levels and disease intensity, Clin Exp Dermatol 25 (2000), 643-647.

[3] N.Y. el Barnawi, A.S. Giasuddin, M.M. Ziu and M. Singh, Serum cytokine levels in psoriasis vulgaris, $\mathrm{Br} \mathrm{J}$ Biomed Sci 58 (2001), 40-44.

[4] H. Takahashi, H. Tsuji, Y. Hashimoto, A. Ishida-Yamamoto and H. Iizuka, Serum cytokines and growth factor levels in Japanese patients with psoriasis, Clin Exp Dermatol 35 (2010), 645-649.

[5] O. Arican, M. Aral, S. Sasmaz and P. Ciragil, Serum levels of TNF- $\alpha$, IFN- $\gamma$, IL-6, IL-8, IL-12, IL-17, and IL-18 in patients with active psoriasis and correlation with disease severity, $\mathrm{Me}$ diators Inflamm 2005 (2005), 273-279.

[6] T. Kawashima, [Analysis of immunological mechanisms in the pathogenesis of generalized pustular psoriasis], Hokkaido Igaku Zasshi 74 (1999), 67-76. (in Japanese)

[7] S. Nakamura, Y. Hashimoto, S. Igawa, M. Kajino, K. Nishi, H. Takahashi, T. Mizumoto and H. Iizuka, Childhood generalized pustular psoriasis treated by preprandial ciclosporin administration: serum cytokine pattern during the course of the disease, Clin Exp Dermatol 34 (2009), e1023-1024.

[8] M. Sakashita, T. Yoshimoto, T. Hirota, M. Harada, K. Okubo, Y. Osawa, S. Fujieda, Y. Nakamura, K. Yasuda, K. Nakanishi and M. Tamari, Association of serum interleukin-33 level and the interleukin-33 genetic variant with Japanese cedar pollinosis, Clin Exp Allergy 38 (2008), 1875-1881.

[9] K. Iwatsuki, T. Terui, A. Ozawa, M. Komine, Y. Umezawa, H. Torii, G. Nakanishi, H. Hara, T. Mabuchi, Y. Aoyama and
Y. Kitajima, Guidelines for the Management of Generalized Pustular Psoriasis 2010, Jpn J Dermatol 120 (2010), 815-839. (in Japanese)

[10] M. Terada, H. Tsutsui, Y. Imai, K. Yasuda, H. Mizutani, K. Yamanishi, M. Kubo, K. Matsui, H. Sano and K. Nakanishi, Contribution of IL-18 to atopic-dermatitis-like skin inflammation induced by Staphylococcus aureus product in mice, Proc Natl Acad Sci U S A 103 (2006), 8816-8821.

[11] Y. Haenuki, K. Matsushita, S. Futatsugi-Yumikura, K.J. Ishii, T. Kawagoe, Y. Imoto, S. Fujieda, M. Yasuda, Y. Hisa, S. Akira, K. Nakanishi and T. Yoshimoto, A critical role of IL33 in experimental allergic rhinitis, J Allergy Clin Immunol (2012).

[12] A. Blauvelt, T-helper 17 cells in psoriatic plaques and additional genetic links between IL-23 and psoriasis, $J$ Invest Dermatol 128 (2008), 1064-1067.

[13] E. Toichi, G. Torres, T.S. McCormick, T. Chang, M.A. Mascelli, C.L. Kauffman, N. Aria, A.B. Gottlieb, D.E. Everitt, B. Frederick, C.E. Pendley and K.D. Cooper, An anti-IL-12p40 antibody down-regulates type 1 cytokines, chemokines, and IL-12/IL-23 in psoriasis, J Immunol 177 (2006), 4917-4926.

[14] A. Steensberg, C.P. Fischer, C. Keller, K. Moller and B.K. Pedersen, IL-6 enhances plasma IL-1ra, IL-10, and cortisol in humans, Am J Physiol Endocrinol Metab 285 (2003), E433437.

[15] B. Dugas, S. Charbonnier, M. Baarine, K. Ragot, D. Delmas, F. Menetrier, J. Lherminier, L. Malvitte, T. Khalfaoui, A. Bron, C. Creuzot-Garcher, N. Latruffe and G. Lizard, Effects of oxysterols on cell viability, inflammatory cytokines, VEGF, and reactive oxygen species production on human retinal cells: cytoprotective effects and prevention of VEGF secretion by resveratrol, Eur J Nutr 49 (2010), 435-446.

[16] N. Mizuki, A. Meguro, M. Ota, S. Ohno, T. Shiota, T. Kawagoe, N. Ito, J. Kera, E. Okada, K. Yatsu, Y.W. Song, E.B. Lee, N. Kitaichi, K. Namba, Y. Horie, M. Takeno, S. Sugita, M. Mochizuki, S. Bahram, Y. Ishigatsubo and H. Inoko, Genomewide association studies identify IL23R-IL12RB2 and IL10 as Behcet's disease susceptibility loci, Nat Genet 42 (2010), 703-706.

[17] M.A. Lowes, A.M. Bowcock and J.G. Krueger, Pathogenesis and therapy of psoriasis, Nature 445 (2007), 866-873.

[18] M. Murakami, E. Hagforsen, V. Morhenn, A. IshidaYamamoto and H. Iizuka, Patients with palmoplantar pustulosis have increased IL-17 and IL-22 levels both in the lesion and serum, Exp Dermatol 20 (2011), 845-847.

[19] S.B. Yilmaz, N. Cicek, M. Coskun, O. Yegin and E. Alpsoy, Serum and tissue levels of IL-17 in different clinical subtypes of psoriasis, Arch Dermatol Res 304 (2012), 465-469.

[20] Y.H. Lo, K. Torii, C. Saito, T. Furuhashi, A. Maeda and A. Morita, Serum IL-22 correlates with psoriatic severity and serum IL-6 correlates with susceptibility to phototherapy, $J$ Dermatol Sci 58 (2010), 225-227. 


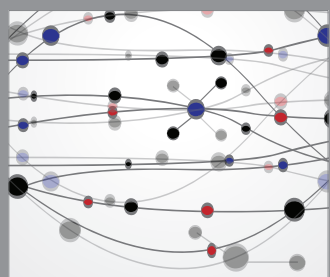

The Scientific World Journal
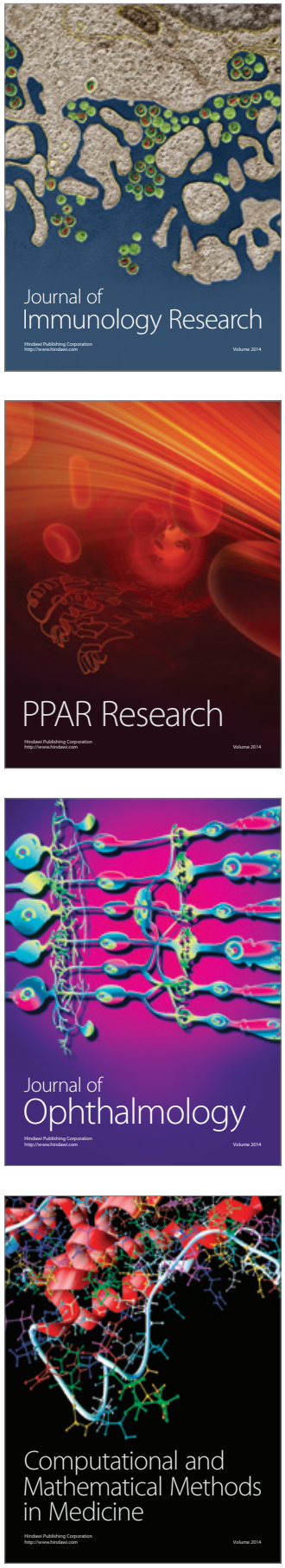

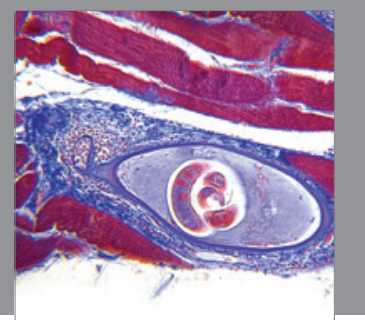

Gastroenterology

Research and Practice
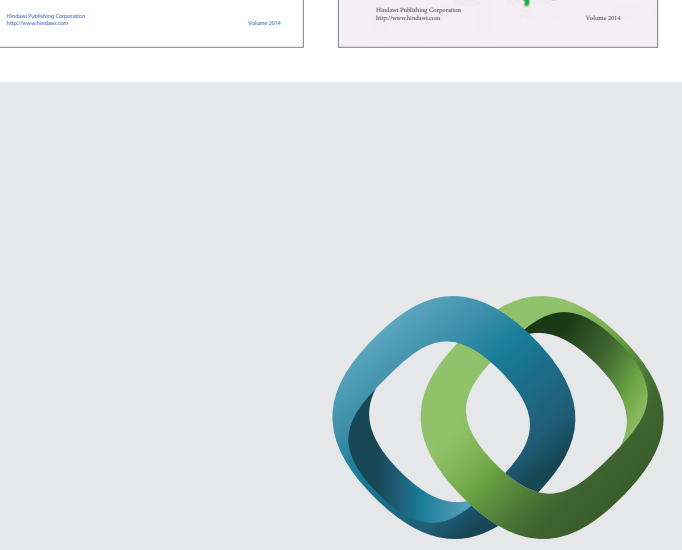

\section{Hindawi}

Submit your manuscripts at

http://www.hindawi.com
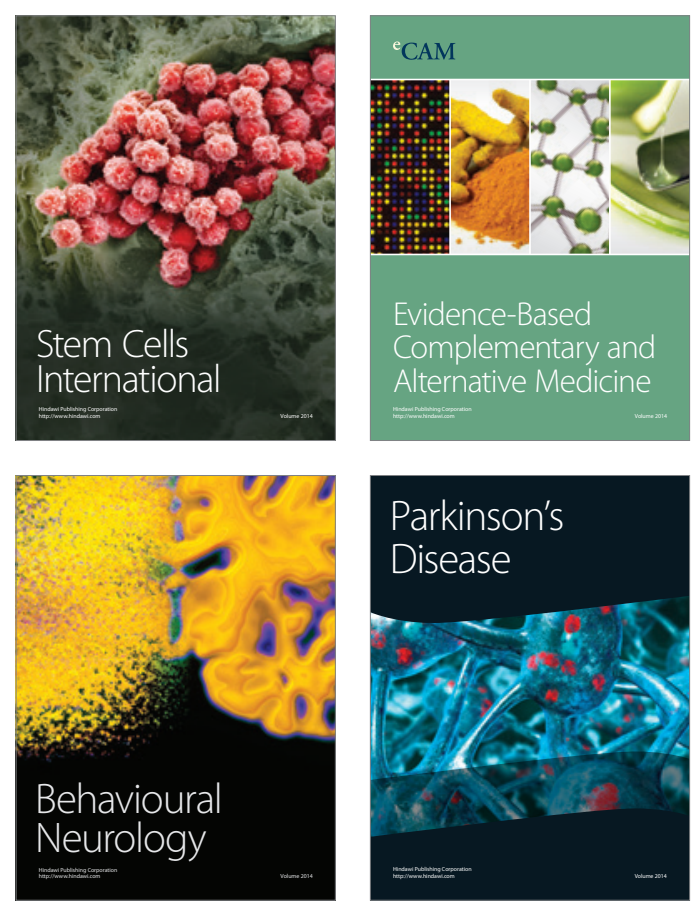

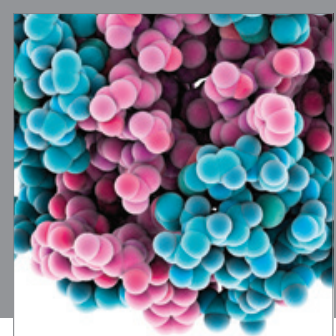

Journal of
Diabetes Research

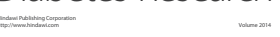

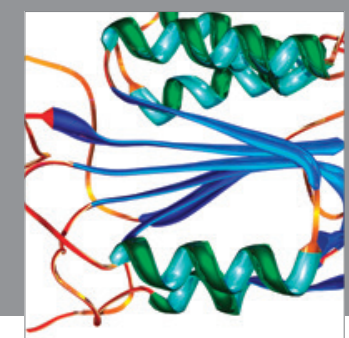

Disease Markers
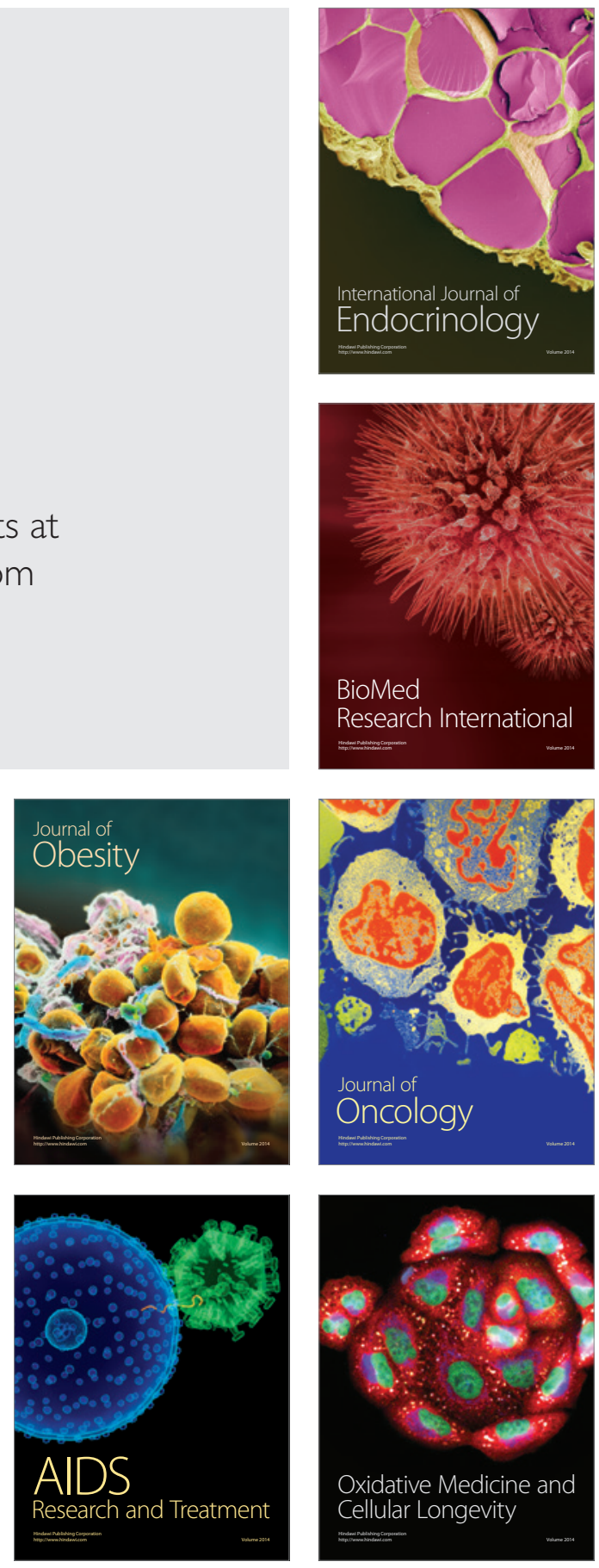